\title{
Five-Dimensional Space-Times with a Variable Gravitational and Cosmological Constant
}

\author{
Sanjay Oli \\ Department of Applied Mathematics, R K G Institute of Technology, Ghaziabad 203201, India \\ Correspondence should be addressed to Sanjay Oli; sanjayoli@rediffmail.com
}

Received 6 September 2013; Accepted 24 November 2013; Published 2 January 2014

Academic Editor: Kazuharu Bamba

Copyright (C) 2014 Sanjay Oli. This is an open access article distributed under the Creative Commons Attribution License, which permits unrestricted use, distribution, and reproduction in any medium, provided the original work is properly cited.

\begin{abstract}
We have presented cosmological models in five-dimensional Kaluza-Klein space-time with a variable gravitational constant $(G)$ and cosmological constant $(\Lambda)$. We have investigated Einstein's field equations for five-dimensional Kaluza-Klein space-time in the presence of perfect fluid with time dependent $G$ and $\Lambda$. A variety of solutions have been found in which $G$ increases and $\Lambda$ decreases with time $t$, which matches with current observation. The properties of fluid and kinematical parameters have been discussed in detail.
\end{abstract}

\section{Introduction}

The observational analysis of High-Redshift Type Ia Supernova and Supernova Cosmological Project [1-5] provided a wealth of information about our universe. These observational analyses imply that a positive cosmological constant of order $\left(\mathrm{Gh} / \mathrm{c}^{3} \approx 10^{-123}\right)$ may dominate the total energy density in the universe and that the expansion of the universe is accelerating [6].

Higher-dimensional cosmological models play a vital role in many aspects of early stage of cosmological problems, one of the frontier areas of research to unify gravity with other forces in nature. The study of higher-dimensional space-time provides an idea that our universe was much smaller at early stage of evolution than observed today. The detection of extra dimensions in current experiments is beyond those four dimensions observed so far.

Over the past few years, a lot of attention was received on cosmological models in which space-time has more than five dimensions. The field of cosmology has been highly enriched by the Kaluza-Klein theory $[7,8]$, in which they have shown that gravitation and electromagnetism could be unified in a single geometrical structure. Chodos and Detweiler obtained a higher-dimensional cosmological model in which an extra dimension contracts and indicates that this contraction of extra dimension is a consequence of cosmological evolution
[9]. Guth and Alvarez and Gavela noticed that during contraction process extra dimensions produce massive amount of entropy, which provides an alternative resolution to the flatness and horizon problems as compared to the usual inflationary scenario [10, 11]. A number of authors [12-22] obtained the solutions of Einstein's field equations for higherdimensional space-times containing a variety of matter fields. In their analysis, some authors have shown that there is an expansion of the four-dimensional space-times while the fifth dimension contracts or remains constant.

The concept of a variable gravitational constant $G$ was first proposed by Dirac in 1937 [23]. In 1985, Lau proposed modifications linking the variation of $G$ with that of $\Lambda$ in the framework of general relativity [24]. This modification allows us the form of Einstein's field equations formally unchanged since a variation in $\Lambda$ is accompanied by a variation of $G$. The present approach is noncovariant, and Einstein's field equations cannot be obtained from a Hamiltonian. This approach solves many cosmological problems, namely, cosmological constant problem, initial singularity problem, and inflationary universe scenario.

A limited number of authors studied cosmological models in higher-dimensional space-time with variable gravitational constant " $G$ " and cosmological constant " $\Lambda$ " [25-29]. Such five-dimensional space-times are also widely studied in the braneworld scenario and are useful to investigate 
([30] and references therein) the geodesic deviation and the kinematics of geodesic flows in the background of such geometries.

Motivated by the above investigation, we investigate physically sound cosmological models in five-dimensional Kaluza-Klein space-times with variable $G$ and $\Lambda$ in this paper. For this purpose, we solve Einstein's field equations for five-dimensional Kaluza-Klein space-time in the presence of perfect fluid with time dependent $G$ and $\Lambda$.

\section{Einstein's Field Equations for Kaluza-Klein Space-Times}

The five-dimensional Kaluza-Klein space-time is described by [29]

$$
d s^{2}=-d t^{2}+X^{2}(t)\left(d x^{2}+d y^{2}+d z^{2}\right)+A^{2}(t) d \psi^{2} .
$$

The Einstein's field equations with time-dependent gravitational and cosmological "constants" are given by

$$
R_{i j}-\frac{1}{2} g_{i j} R=-8 \pi G(t) T_{i j}-\Lambda(t) g_{i j}
$$

Energy momentum tensor for a one-fluid source is

$$
T_{i j}=(p+\rho) u_{i} u_{j}-p g_{i j}
$$

with

$$
g^{i j} u_{i} u_{j}=1,
$$

where $\rho$ is the energy density and $p$ is the isotropic pressure of the fluid.

From WMAP (Wilkinson Microwave Anisotropy Probe) data present, overall energy density of the universe is very low, roughly $9.9 \times 10^{-30}$ grams per cubic centimeter. This mass-energy appears to consist of $73 \%$ dark energy, $23 \%$ cold dark matter, and $4 \%$ ordinary visible matter. The gravitational constant $(G)$ is given by approximately $6.67 \times 10^{-11} \mathrm{~m} / \mathrm{kg}^{2}$, and in terms of Planck units, as a natural dimensionless value, the cosmological constant $(\Lambda)$ is on the order of $10^{-123}$.

$u_{i}$ is the five-velocity of the time-like vector satisfying (4). The off-diagonal equations of (2) together with energy conditions $p+\rho \geq 0$ and $\rho \geq 0$ imply that

$$
u_{i}=(0,0,0,0,1) .
$$

Using (2), (3), and (5), surviving field equations (1) are

$$
\begin{gathered}
3 \frac{\dot{X} \dot{A}}{X A}+3 \frac{\dot{X}^{2}}{X^{2}}=8 \pi G \rho+\Lambda, \\
3 \frac{\ddot{X}}{X}+3 \frac{\dot{X}^{2}}{X^{2}}=-8 \pi G p+\Lambda, \\
2 \frac{\ddot{X}}{X}+\frac{\ddot{A}}{A}+\frac{\dot{X}^{2}}{X^{2}}+2 \frac{\dot{X} \dot{A}}{X A}=-8 \pi G p+\Lambda,
\end{gathered}
$$

where the dots denote differentiation with respect to $t$. The usual conservation law for Einstein's field equation $T_{; j}^{i j}=0$ (; i.e., semicolon, denotes covariant divergence) implies

$$
\dot{\rho}+\left(3 \frac{\dot{X}}{X}+\frac{\dot{A}}{A}\right)(\rho+p)=0
$$

In Einstein's theory, the principle of equivalence requires that $G$ and $\Lambda$ do not enter the equation of motion of particles and photons; that is, only $g_{i j}$ must enter them. The vanishing of the covariant divergence of the Einstein tensor in (2) and using (9), we obtain

$$
\dot{\Lambda}=-8 \pi \dot{G} \rho
$$

These are four equations ((6)-(9) or (10)) in six unknowns. Thus to get a solution, we require two additional relations. These relations may be taken to involve field variables as well as physical variables. In the following sections, we will explore the possibility of finding physically meaningful solutions of the field equations subject to specified geometrical and physical conditions.

\section{Solutions of Field Equations}

One of the relations is the equation of state

$$
p=w \rho, \quad-1 \leq w \leq 1 .
$$

From (7) and (8) an equation connecting $X$ and $A$ is obtained:

$$
\frac{\ddot{X}}{X}-\frac{\ddot{A}}{A}+2 \frac{\dot{X}^{2}}{X^{2}}-2 \frac{\dot{X} \dot{A}}{X A}=0 .
$$

Integrating (12), we obtain

$$
\frac{\dot{X}}{X}-\frac{\dot{A}}{A}=\frac{k}{A X^{3}},
$$

where $k$ is an integration constant. Using (13) and the transformation

$$
A X^{3}=U
$$

where $U$ is the function of time $t, X$ and $A$ are expressed in terms of $U$ as follows:

$$
X=\beta U^{(1 / 4)} e^{(k / 4) \int(d t / U)}, \quad A=\delta U^{(1 / 4)} e^{-(3 k / 4) \int(d t / U)},
$$

where $\beta$ and $\delta$ are integration constants. Using (15), the resulting solution of field equations ((6)-(11)) can be expressed in terms of $U$ as follows:

$$
\begin{gathered}
\rho=\alpha U^{-(1+w)}, \\
\Lambda=\frac{3\left[2 U \ddot{U}+(w-1)\left(\dot{U}-k^{2}\right)\right]}{8(1+w) U^{2}}, \\
G=\frac{3 U^{(w-1)}\left[\dot{U}^{2}-U \ddot{U}-k^{2}\right]}{32 \pi(1+w) \alpha} .
\end{gathered}
$$

The cosmological parameters are given by

$$
\theta=\frac{\dot{U}}{U}, \quad \sigma^{2}=\frac{3 k^{2}}{8 U^{2}}, \quad q=\frac{\left[2 \dot{U}^{2}-3 U \ddot{U}\right]}{\dot{U}^{2}} .
$$


The jerk parameter in cosmology is defined as the dimensionless third derivative of the field parameters with respect to cosmic time; we can write it as

$$
j(t)=q+2 q^{2}-\frac{\dot{q}}{H} .
$$

Here, we have noticed that $\omega^{2}=0$ for all cases mentioned below. We come across three types of situation depending upon the parameter $U$.

Case I. $U=c=$ constant. In this case $X=\beta^{\prime} e^{\left(k^{\prime} t / 4\right)}, A=$ $\delta^{\prime} e^{-\left(3 k^{\prime} t / 4\right)}, \rho=\alpha c^{-(1+w)}, G=-\left(3 c^{(w-1)} k^{2} / 32 \pi(1+\right.$ $w) \alpha), \Lambda=\left(3(1-w) k^{\prime 2} / 8(1+w)\right), \theta=0, \sigma=\left(3 k^{\prime 2} / 8\right)$, and $q$ is not defined, where $\beta^{\prime}=\beta c^{(1 / 4)}, \delta^{\prime}=\delta c^{(1 / 4)}$, and $k^{\prime}=k / c$. Here $\rho, G, \Lambda$, and $\sigma$ are constant and for $k=0, G$, $\Lambda$, and $\sigma$ become significant. For $k>0, X$ increases and the extra dimension $A$ decreases exponentially with time, and we retrieved the solution obtained by Arbab [31].

Case II. $U=t$. In this case $X=\beta t^{((1+k) / 4)}, A=\delta t^{((1-3 k) / 4)}$, $\rho=\alpha t^{-(1+w)}, G=-\left(3 t^{(w-1)}\left(k^{2}-1\right) / 32 \pi(1+w) \alpha\right), \Lambda=(3(1-$ $\left.w)\left(k^{2}-1\right) / 8(1+w) t^{2}\right), \theta=(1 / t), \sigma^{2}=\left(3 k^{2} / 8 t^{2}\right)$, and $q=2$. Putting $k=((1-n) /(n+3))$, we retrieved the solution obtained by Baysal and Yilmaz [29].

Case III. $U=t^{n}$. In this case, $X=\beta t^{(n / 4)} e^{\left(k t^{(1-n)} / 4(1-n)\right)}, A=$ $\delta t^{(n / 4)} e^{-\left(3 k t^{(1-n)} / 4(1-n)\right)}, \rho=\alpha t^{-n(1+w)}, G=\left(3 t^{n(w-1)}\left[n t^{2(n-1)}-\right.\right.$ $\left.\left.k^{2}\right] / 32 \pi(1+w) \alpha\right), \quad \Lambda=(3 / 8(1+w))\left[\left(\left((1+w) n^{2}-2 n\right) / t^{2}\right)-\right.$ $\left.\left((w-1) k^{2} / t^{2 n}\right)\right], \theta=n / t, \sigma^{2}=\left(3 k^{2} / 8 t^{2 n}\right)$, and $q=((3-n) / n)$, where $w, n \neq 1, j(t)=1+\left(18 / n^{2}\right)-(9 / n)$.

For $n=1.65$, the jerk parameter reduces to value $j \approx 2.16$. This value is equivalent to the value obtained from the combination of the three kinematical data sets: the gold sample of Type Ia supernova [4], the SNIa data from the SNLS project [32] and the X-ray galaxy cluster distance measurement [33].

For $n>0, \theta$ shows the condition of expansion. The expansion scalar $\theta \rightarrow 0$ as $\rightarrow \infty$; that is, the universe is expanding as time $t$ increases, and the rate of expansion is slowing down with increasing time $t$. Also, for $n>0$, the shear tends to zero as time increases. For $0<n<1$, the ratio $\sigma / \theta$ does not drop to zero as $t \rightarrow \infty$, which implies that shear does not tend to zero faster than the expansion scalar. This indicates that the model presented above remains anisotropic and our solution represents the early stage of evolution of the universe. For $n>1$, the ratio $\sigma / \theta$ tends to zero as $t \rightarrow \infty$, which implies that the model isotropies as time increases. The deceleration parameter $q$ is negative for $n>3$, shows the inflationary character of the model in early stage of the universe.

At the present epoch, the extra dimension has no significant effect on the evolution of the universe. The extra dimension must have become insignificant with increasing time $t$ after the creation. For our model, $k>0$ and $0<$ $n<1$, the dimension $X$ increases and extra dimension $A$ decreases exponentially with time $t$. This indicates that the extra dimension becomes insignificant as $t \rightarrow \infty$, and we are left with the real four-dimensional universe.

For $k>0$ and $0<n<1, \Lambda$ approaches to $-\infty$ as $t \rightarrow 0$ and becomes insignificant for $t=$ $t^{*}=\left[\left(2 n-(1+w) n^{2}\right) /\left((1-w) k^{2}\right)\right]^{(1 /(2(1-n)))}$, where $w \neq 1$. For $t>t^{*}, \Lambda$ initially increases till it attains its maximum value and decreases monotonically afterwards. For $w=1$, the cosmological constant $\Lambda \propto t^{-2}$ is a nonnegative decreasing function of time $t$.

The gravitational constant $G$ approaches to $\infty$ as $t \rightarrow 0$. For $0<t<t_{*}, G$ is nonnegative decreasing function of time $t$ and becomes zero for $t=t_{*}=\left[n / k^{2}\right]^{(1 /(2(1-n)))} . G<0$ for $t>t_{*}$ and decreases with time $t$.

The density $\rho$ decreases with time $t$, and its value depends upon the value of the parameters $n$ and $w$. For $w=-1, \Lambda$ and $G$ diverge and the density $\rho$ is constant for all $t$. In this phase, the universe is static in nature. However, in order to investigate the evolution of kinematical quantities, namely, expansion scalar $\theta$, shear $\sigma$, and rotation $\omega$, as mentioned above, one needs to solve the corresponding Raychaudhuri equations $[34,35]$.

\section{Conclusion}

In the forgoing sections, we have presented five-dimensional Kaluza-Klein cosmological models with a variable gravitational constant $G$ and cosmological constant $\Lambda$. We obtained the solution of Einstein's field equations by assuming the transformation $A X^{3}=U$ and equation of state $p=w \rho$. We investigated three different cases giving the particular value of $U$ as constants, $t$ and $t^{n}$. We rediscovered the solution obtained [31] for $U=$ constant. The cosmological solutions of Baysal and Yilmaz [29] are retrieved from our model for $k=((1-n) /(n+3))$.

For $0<n<1$, we obtain class of cosmological models, where gravitational constant $G$ decreases with time $t$. Also, the cosmological constant $\Lambda$ decreases with time $t$, which is supported by results from recent supernovae Ia observations and matches its natural units. This supports views that favor the dependence $\Lambda \propto t^{-2}$, as expressed by several authors ([34] and see references therein). In our models, the ratio $\sigma / \theta$ does not drop to zero as $t \rightarrow \infty$, which implies that the model presented above remains anisotropic and our solution represents the early stage of evolution of the universe.

\section{Conflict of Interests}

The author declares that there is no conflict of interests regarding the publication of this paper.

\section{References}

[1] P. M. Garnavich, R. P. Kirshner, P. Challis et al., "Constraints on cosmological models from Hubble Space Telescope observations of high-z supernovae," Astrophysical Journal Letters, vol. 493, no. 2, pp. L53-L58, 1998. 
[2] S. Perlmutter, G. Aldering, M. Della Valle et al., "Discovery of a supernova explosion at half the age of the Universe," Nature, vol. 391, no. 6662, pp. 51-54, 1998.

[3] S. Perlmutter, G. Aldering, G. Goldhaber et al., "Measurements of $\Omega$ and $\Lambda$ from 42 high-redshift supernovae," The Astrophysical Journal, vol. 517, no. 2, pp. 565-586, 1999.

[4] A. G. Riess, A. V. Filippenko, P. Challis et al., "Observational evidence from supernovae for an accelerating universe and a cosmological constant," Astronomical Journal, vol. 116, no. 3, pp. 1009-1038, 1998.

[5] B. P. Schmidt, N. B. Suntzeff, M. M. Phillips et al., "The high-Z supernova search: measuring cosmic deceleration and global curvature of the universe using type Ia supernovae," Astrophysical Journal Letters, vol. 507, no. 1, pp. 46-63, 1998.

[6] V. Sahni and A. Staobinsky, "The case for a positive cosmological $\Lambda$-term," International Journal of Modern Physics D, vol. 9, pp. 373-443, 2000.

[7] T. Kaluza, Zum Unitätsproblem der Physik, Sitzungsberichte der Preussischen Akademie der Wissenschaften, Berlin, Germany, 1921.

[8] O. Klein, "Quantentheorie und fünfdimensionale Relativitätstheorie," Zeitschrift für Physik, vol. 37, pp. 895-906, 1926.

[9] A. Chodos and S. Detweiler, "Where has the fifth dimension gone?” Physical Review D, vol. 21, no. 8, pp. 2167-2170, 1980.

[10] A. H. Guth, "Inflationary universe: a possible solution to the horizon and flatness problems," Physical Review D, vol. 23, no. 2, pp. 347-356, 1981.

[11] E. Alvarez and M. B. Gavela, "Entropy from extra dimensions," Physical Review Letters, vol. 51, no. 10, pp. 931-934, 1983.

[12] P. G. O. Freund, "Kaluza-Klein cosmologies," Nuclear Physics B, vol. 209, no. 1, pp. 146-156, 1982.

[13] T. Appelquist and A. Chodos, "Quantum effects in Kaluza-Klein theories," Physical Review Letters, vol. 50, no. 3, pp. 141-145, 1983.

[14] S. Randjbar-Daemi, A. Salam, and J. Strathdee, "On KaluzaKlein cosmology," Physics Letters B, vol. 135, no. 5-6, pp. 388392, 1984.

[15] F. Rahaman, S. Chakraborty, N. Begum, M. Hossain, and M. Kalam, "Bianchi-IX string cosmological model in Lyra geometry," Fizika B, vol. 11, pp. 57-62, 2002.

[16] F. Rahaman, B. C. Bhui, and B. Bhui, "Cosmological model with a viscous fluid in a Kaluza-Klein metric," Astrophysics and Space Science, vol. 301, no. 1-4, pp. 47-49, 2006.

[17] G. P. Singh, R. V. Deshpande, and T. Singh, "Higherdimensional cosmological model with variable gravitational constant and bulk viscosity in lyra geometry," Pramana, vol. 63, no. 5, pp. 937-945, 2004.

[18] G. S. Khadekar, A. Pradhan, and M. R. Molaei, "Higher dimensional dust cosmological implications of a decay law for the a term: expressions for some observable quantities," International Journal of Modern Physics D, vol. 15, no. 1, pp. 95105, 2006.

[19] I. Yilmaz and A. A. Yavuz, "Higher-dimensional cosmological models with strange quark matter," International Journal of Modern Physics D, vol. 15, no. 4, pp. 477-483, 2006.

[20] G. Mohanty, K. L. Mahanta, and R. R. Sahoo, "Non-existence of five dimensional perfect fluid cosmological model in Lyra manifold," Astrophysics and Space Science, vol. 306, no. 4, pp. 269-272, 2006.

[21] A. Pradhan, G. S. Khadekar, M. K. Mishra, and S. Kumbhare, "Higher dimensional strange quark matter coupled to the string cloud with electromagnetic field admitting one parameter group of conformal motion," Chinese Physics Letters, vol. 24, no. 10, pp. 3013-3016, 2007.

[22] K. D. Purohit and Y. Bhatt, "Static extra dimension and acceleration of the universe," International Journal of Theoretical Physics, vol. 50, no. 5, pp. 1417-1423, 2011.

[23] P. A. M. Dirac, “The cosmological constants," Nature, vol. 139, no. 3512, article 323, 1937.

[24] Y. K. Lau, "The large number hypothesis and Einstein's theory of gravitation," Australian Journal of Physics, vol. 38, pp. 547-553, 1985.

[25] A. Abdussattar and R. G. Vishwakarma, "Some RobertsonWalker models with variable G and $\Lambda$," Australian Journal of Physics, vol. 50, no. 5, pp. 893-901, 1997.

[26] S. Chakraborty and A. Ghosh, "Generalized scalar tensor theory in four and higher dimension," International Journal of Modern Physics D, vol. 9, no. 5, pp. 543-549, 2000.

[27] F. Rahaman and J. K. Bera, "Higher dimensional cosmological model in Lyra geometry," International Journal of Modern Physics D, vol. 10, no. 5, pp. 729-733, 2001.

[28] G. S. Khadekar, G. L. Kondawar, V. Kamdi, and C. Ozel, "Early viscous universe with variable cosmological and gravitational constants in higher dimensional space time," International Journal of Theoretical Physics, vol. 47, no. 11, pp. 3057-3074, 2008.

[29] H. Baysal and I. Yilmaz, "Five-dimensional cosmological model with variable G and $\Lambda$," Chinese Physics Letters, vol. 24, no. 8, article 009, pp. 2185-2188, 2007.

[30] S. Ghosh, S. Kar, and H. Nandan, "Confinement of test particles in warped spacetimes," Physical Review D, vol. 82, no. 2, Article ID 024040, 2010.

[31] A. I. Arbab, "Comment on 'five-dimensional cosmological model with variable $\mathrm{G}$ and $\Lambda$,' Chinese Physics Letters, vol. 25, no. 1, p. 351, 2008.

[32] P. Astier, J. Guy, N. Regnault et al., "The Supernova Legacy Survey: measurement of $\Omega_{M}, \Omega_{\Lambda}$ and $w$ from the first year data set ," Astronomy \& Astrophysics, vol. 447, no. 1, pp. 31-48, 2006.

[33] D. Rapeui, S. W. Allen, M. A. Amin, and R. D. Blandford, "A kinematical approach to dark energy studies," Monthly Notices of the Royal Astronomical Society, vol. 375, no. 4, pp. 1510-1520, 2007.

[34] A. Dasgupta, H. Nandan, and S. Kar, "Kinematics of deformable media," Annals of Physics, vol. 323, no. 7, pp. 1621-1643, 2008.

[35] A. Dasgupta, H. Nandan, and S. Kar, "Kinematics of geodesic flows in stringy black hole backgrounds," Physical Review D, vol. 79, no. 12, Article ID 124004, 2009. 

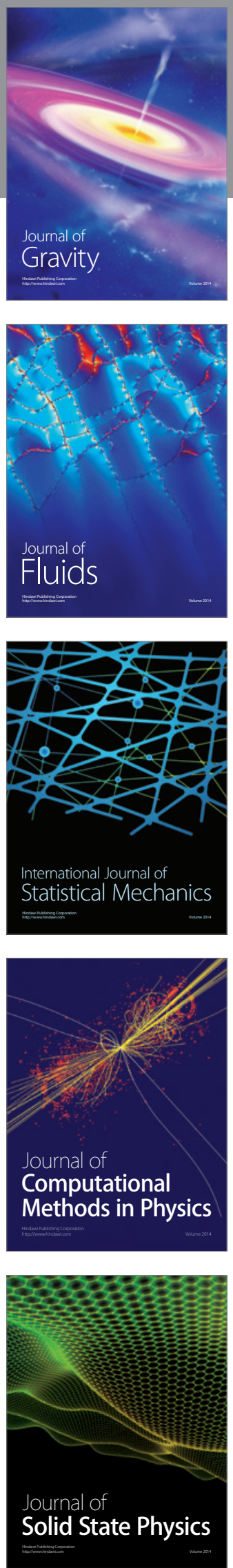

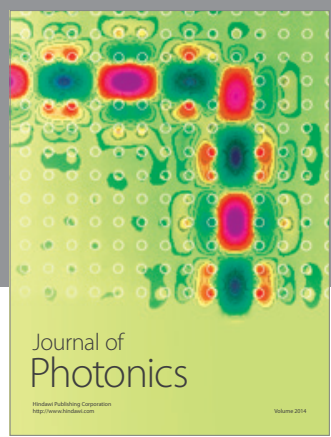

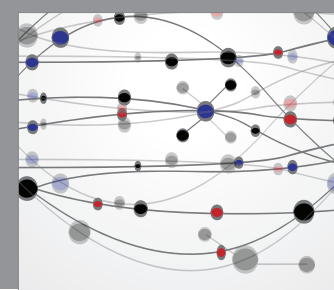

The Scientific World Journal

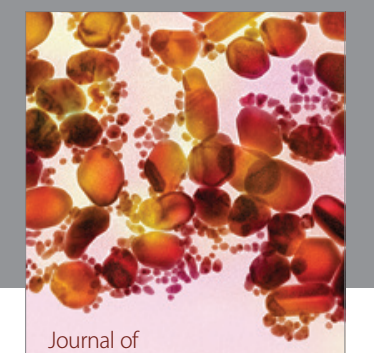

Soft Matter
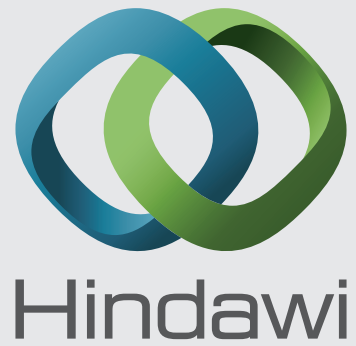

Submit your manuscripts at

http://www.hindawi.com
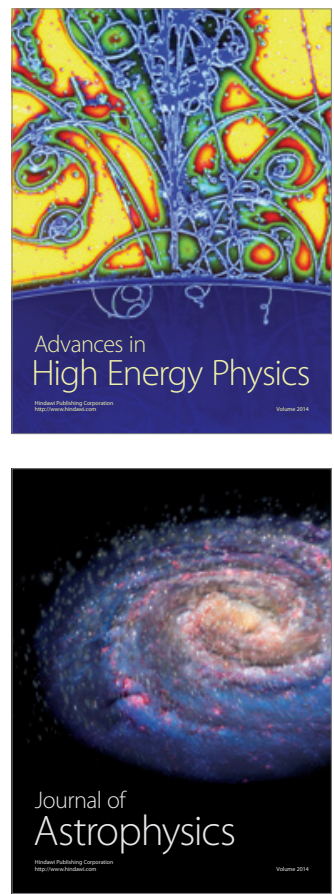
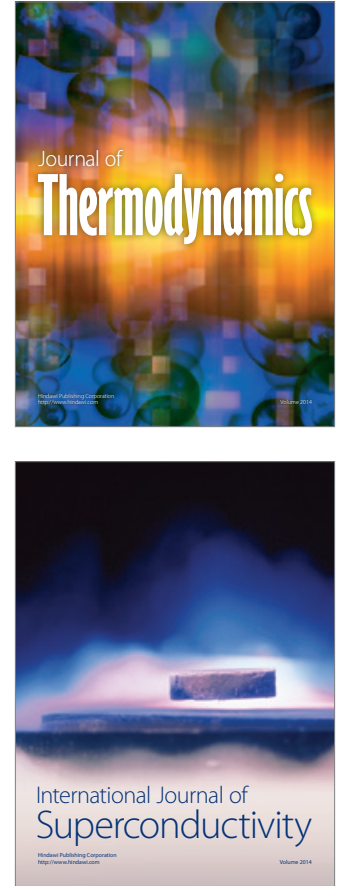
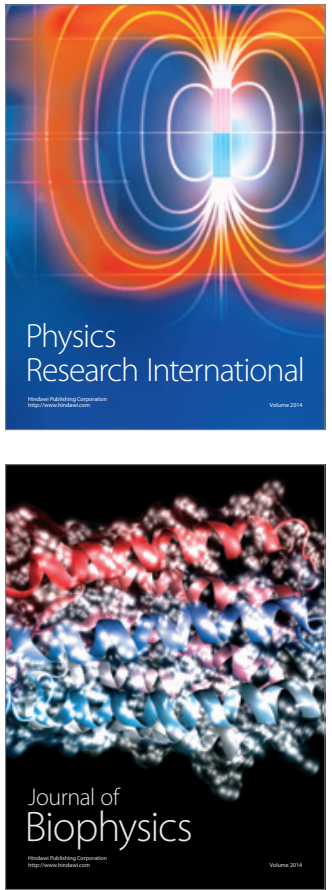
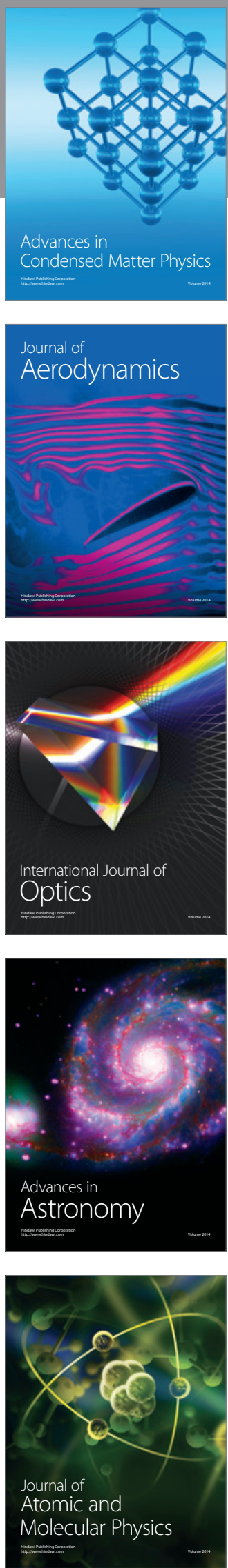